STRUCTURAL BIOLOGY

ISSN 2059-7983
Received 12 May 2015

Accepted 15 December 2015

₹ These authors contributed equally to this work.

$\S$ Present address: National Center for Protein Sciences, State Key Laboratory of Molecular Biology, Institute of Biochemistry and Cell Biology, Shanghai Institutes for Biological Sciences, Chinese Academy of Sciences, Shanghai, People's Republic of China.

Keywords: native SAD phasing; anomalous signal; data-collection strategy; complex; membrane protein.

Supporting information: this article has supporting information at journals.iucr.org/d

\section{Data-collection strategy for challenging native SAD phasing}

\author{
Vincent Olieric, ${ }^{a *} \ddagger$ Tobias Weinert, ${ }^{\mathrm{a}} \ddagger$ Aaron D. Finke, ${ }^{\mathrm{a}}$ Carolin Anders, ${ }^{\mathrm{b}}$ Dianfan \\ $\mathrm{Li}^{\mathrm{c}}{ }^{\mathrm{S}}$ Natacha Olieric, ${ }^{\mathrm{d}}$ Camelia N. Borca, ${ }^{\mathrm{a}}$ Michel O. Steinmetz, ${ }^{\mathrm{d}}$ Martin Caffrey, ${ }^{\mathrm{c}}$ \\ Martin Jinek ${ }^{\mathrm{b}}$ and Meitian Wang ${ }^{\mathrm{a}}$
}

\footnotetext{
${ }^{a}$ Swiss Light Source, Paul Scherrer Institut, Villigen PSI, Switzerland, 'bepartment of Biochemistry, University of Zurich, Zurich, Switzerland, ' ${ }^{\mathrm{c} M e m b r a n e}$ Structural and Functional Group, Schools of Medicine and Biochemistry and Immunology, Trinity College, Dublin, Ireland, and 'Laboratory of Biomolecular Research, Department of Biology and Chemistry, Paul Scherrer Institut, Villigen PSI, Switzerland. *Correspondence e-mail: vincent.olieric@psi.ch
}

Recent improvements in data-collection strategies have pushed the limits of native SAD (single-wavelength anomalous diffraction) phasing, a method that uses the weak anomalous signal of light elements naturally present in macromolecules. These involve the merging of multiple data sets from either multiple crystals or from a single crystal collected in multiple orientations at a low X-ray dose. Both approaches yield data of high multiplicity while minimizing radiation damage and systematic error, thus ensuring accurate measurements of the anomalous differences. Here, the combined use of these two strategies is described to solve cases of native SAD phasing that were particular challenges: the integral membrane diacylglycerol kinase (DgkA) with a low Bijvoet ratio of $1 \%$ and the large $200 \mathrm{kDa}$ complex of the CRISPRassociated endonuclease (Cas9) bound to guide RNA and target DNA crystallized in the low-symmetry space group $C 2$. The optimal native SAD data-collection strategy based on systematic measurements performed on the $266 \mathrm{kDa}$ multiprotein/multiligand tubulin complex is discussed.

\section{Introduction}

Native SAD (single-wavelength anomalous diffraction) is a de novo macromolecular structure-determination method in which the phase problem is solved by exploiting the anomalous diffraction signal of light atoms present in the crystal (for a review of anomalous diffraction, see Hendrickson, 2014). Unlike other de novo methods, structure solution by native SAD does not require the incorporation of exogenous heavy atoms, which can be tedious and can lead to nonisomorphism. However, native SAD phasing has its own challenges. Firstly, the absorption edge, where anomalous scattering is maximized, is below $2.5 \mathrm{keV}$ ( $>5 \AA$ ) for elements such as sulfur and phosphorus, which is beyond the reach of most current macromolecular crystallography (MX) synchrotron beamlines. Data collection is also hindered by sample and air absorption of X-rays at such low energies. Thus, native SAD data collection is usually performed at 'compromise'

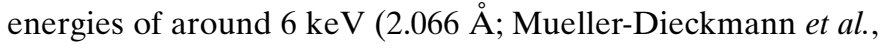
2005; Liu et al., 2014; Weinert et al., 2015) to maximize the anomalous signal while minimizing absorption. Alternatively, home sources, which typically operate with $\mathrm{Cu} K \alpha(1.54 \AA)$ or $\mathrm{Cr} K \alpha \quad(2.29 \AA)$ radiation, can also be used for such measurements. Secondly, because data collection is performed far from the absorption edges of these light elements, the resulting anomalous signal is small and accurate measurements of the reflection intensities and their differences are 
essential. To obtain this level of accuracy, random and systematic errors must be minimized. However, typical datacollection protocols at third-generation synchrotrons, usually performed around a single axis, yield anomalous data that can be adversely affected by radiation damage if insufficient care is given to the X-ray dose delivered to the crystal. Consequently, successful cases of phasing by native SAD remain rare; currently, about 150 native SAD structures have been deposited in the Protein Data Bank (Berman et al., 2000).

Recent advances in data-collection strategies have breathed new life into native SAD phasing (reviewed by Rose et al., 2015). The multi-crystal averaging approach involves merging data sets from statistically equivalent crystals (Liu et al., 2012, 2013), enhancing the anomalous signal-to-noise ratio while minimizing anomalous signal decay owing to radiation damage. The key to successful multi-crystal averaging having enough isomorphous crystals available for data collection. This method is possible because data-merging and dataprocessing algorithms are robust enough to accommodate a large number of distinct crystal data sets. In particularly challenging cases, data sets from as many as 18 (Akey et al., 2014) and 32 (El Omari et al., 2014) separate crystals were required to obtain sufficient anomalous signal for successful phasing. An extreme case is the recent native SAD phasing of lysozyme using serial crystallography data collected at room temperature, where $2^{\circ}$ of data obtained individually from 992 randomly oriented crystals with an average size of $20 \mu \mathrm{m}$ were merged (Huang et al., 2015). A second approach involves collecting many low-dose data sets $\left(<0.5 \mathrm{MGy}\right.$ per $\left.360^{\circ}\right)$ from a single crystal in multiple orientations, mitigating radiation damage while also reducing systematic errors in data collection (Debreczeni et al., 2003; Brockhauser et al., 2013; Weinert et al., 2015; Finke et al., 2016). By varying the crystal orientation, it is possible to measure the same reflections in different diffraction geometries on different areas of the detector and with different sample absorption, thereby reducing systematic error. This approach has proven to be successful for 11 real-life cases, including a $266 \mathrm{kDa}$ multiprotein/multiligand complex, the largest structure solved by native SAD to date (Weinert et al., 2015). The method benefited from instrumentation developments such as a highprecision PRIGo multi-axis goniometer (Waltersperger et al., 2015) and a photon-counting, noise-free pixel-array detector calibrated for low energies (Henrich et al., 2009), both of which were used in the current study.

Despite the many recent improvements in data-collection strategies, difficult cases such as large anomalous scattering substructures, low-symmetry space groups, low diffraction resolution, small crystals, low Bijvoet ratios or some combination thereof still remain a challenge for native SAD phasing. Fortunately, it is possible to combine the two strategies introduced above to extract as much anomalous signal as possible with the highest possible accuracy from as many crystals as needed for successful phasing (Klinke et al., 2015). Here, we present two challenging native SAD cases solved by collecting low-dose data at multiple orientations from three crystals each: the integral membrane diacylglycerol kinase
DgkA (Li et al., 2013; $2 \times 42 \mathrm{kDa}$ asymmetric unit, space group $P 2_{1} 2_{1} 2_{1}, 2.6 \AA$ resolution) and the large Cas9-RNADNA complex (Anders et al., 2014; $200 \mathrm{kDa}$ asymmetric unit, space group $C 2,2.2 \AA$ resolution). In addition, we demonstrate the benefits of these combined low-dose multi-orientation, multi-crystal data-collection strategies using systematic measurements performed on the multiprotein/multiligand tubulin-RB3-tubulin tyrosine ligase complex $\mathrm{T}_{2} \mathrm{R}-\mathrm{TTL}$ (Prota, Bargsten et al., 2013; Weinert et al., 2015; $266 \mathrm{kDa}$ asymmetric unit, space group $P 2_{1} 2_{1} 2_{1}, 2.3 \AA$ resolution).

\section{Materials and methods}

2.1. Crystallographic data collection, data processing and analysis

All experiments were performed on the super-bendingmagnet beamline X06DA (PXIII) at the Swiss Light Source (SLS), Villigen PSI, Switzerland operating at $2.4 \mathrm{GeV}$ with $400 \mathrm{~mA}$ top-up mode. The beamline has a double channel-cut $\mathrm{Si}(111)$ monochromator with an energy resolution of $1.4 \times$ $10^{-4}$ and an elliptical beam of $90 \times 50 \mu \mathrm{m}$ (FWHM). Data were collected at $100 \mathrm{~K}$ with a wavelength of $2.066 \AA(6 \mathrm{keV})$ and a flux of $\sim 1.5 \times 10^{10}$ photons s$^{-1}$. Multi-orientation data collection was carried out at various $\chi$ and $\varphi$ settings of the multi-axis PRIGo goniometer (Waltersperger et al., 2015) and on a PILATUS 2M-F detector (Henrich et al., 2009) operated in shutterless mode at a frame rate of 10 or $20 \mathrm{~Hz}$ and at a sample-to-detector distance of $120 \mathrm{~mm}$. The data were processed using $X D S$ and scaled and merged with XSCALE (Kabsch, 2010). The high-resolution data cutoff was based on the statistical indicators $\mathrm{CC}_{1 / 2}$ and $\mathrm{CC}^{*}$ (Karplus \& Diederichs, 2012). Substructure determination and phasing were performed with $S H E L X C / D / E$ (Sheldrick, 2010) using the HKL2MAP interface (Pape \& Schneider, 2004). The anomalous peak heights were calculated using AnoDe without a resolution cutoff (Thorn \& Sheldrick, 2011). The number of correct sites found with SHELXD was assessed using SITCOM (Dall'Antonia \& Schneider, 2006). Refinement and model map cross-correlation calculations were performed using PHENIX (Adams et al., 2010). X-ray dose was estimated with RADDOSE-3D (Zeldin et al., 2013). The Bijvoet ratio was estimated using the formula

$$
\left(\left|\Delta F^{ \pm}\right|\right) /(|F|)=\left(2 N_{\mathrm{A}} / N_{\mathrm{P}}\right)^{1 / 2} \times\left(f^{\prime \prime} / Z_{\text {eff }}\right),
$$

where $f^{\prime \prime}$ is the imaginary scattering contribution of sulfur $(0.95 \mathrm{e}$ at $6 \mathrm{keV}), N_{\mathrm{A}}$ is the number of anomalous scattering atoms, $N_{\mathrm{P}}$ is the number of non-H atoms in the asymmetric unit, and $Z_{\text {eff }}$ is the effective number of electrons of the average protein atom (6.7; Hendrickson \& Teeter, 1981). Structure figures were prepared and rendered using PyMOL (DeLano, 2002).

2.1.1. DgkA. $P 2{ }_{1} 2_{1} 2_{1}$ crystals of a DgkA mutant (A41C, C46A, I53V, I70L, M96L, V107D, C113A) measuring 100$200 \times 50 \times 20 \mu \mathrm{m}$ (Supplementary Fig. S1a) were grown by the in meso or lipid cubic phase method as described elsewhere (Li et al., 2013), harvested and snap-cooled in liquid 
Table 1

Summary of data collection and statistics.

Values in parentheses are for the last shell.

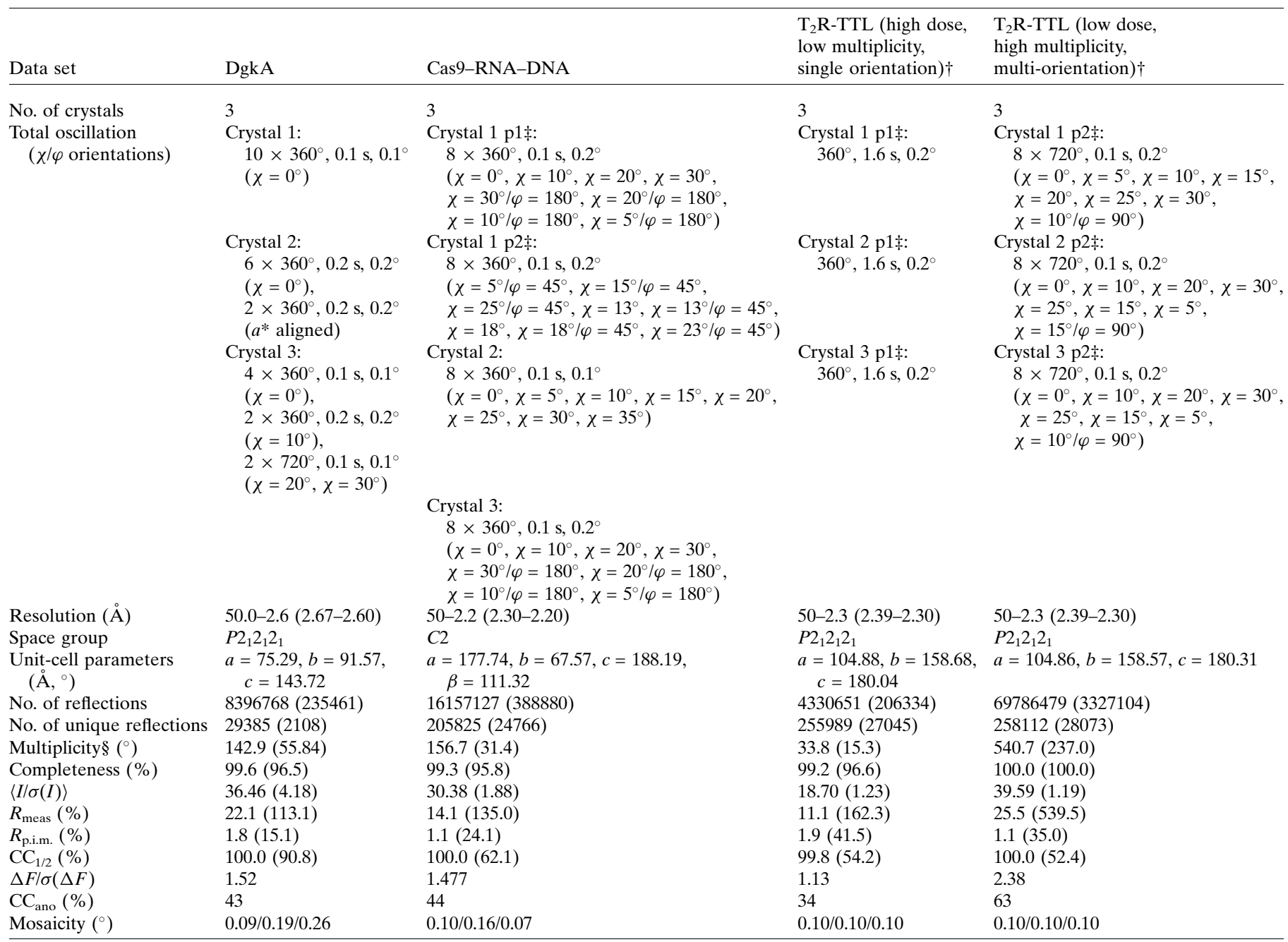

$\dagger$ See Supplementary Data for XDS/XSCALE processing statistics of both merged and individual data sets. $\ddagger$ For crystals that were translated during measurement, the different positions are labelled $\mathrm{p} 1$ and $\mathrm{p} 2$. \& Friedel pairs are counted as merged reflections.

nitrogen. Three crystals were used for data collection (Supplementary Fig. S1a). From the first crystal, which diffracted to $2.6 \AA$ resolution, a total of ten $360^{\circ} \omega$ scans were collected with no change in orientation. The second crystal diffracted to $2.8 \AA$ resolution and a total of eight $360^{\circ} \omega$ scans were collected, including two $360^{\circ} \omega$ scans with the crystal aligned along the $a^{*}$ axis in order to collect Bijvoet pairs simultaneously on the same image. Data for the third crystal, which diffracted to $2.8 \AA$ resolution, were collected in multiple orientations: four $360^{\circ} \omega$ scans at $\chi=0^{\circ}$ and two $360^{\circ} \omega$ scans each at $\chi=10,20$ and $30^{\circ}$ (Table 1 ). At a data-collection speed of $1 \mathrm{deg} \mathrm{s}^{-1}$, the average dose per $360^{\circ} \omega$ scan was estimated to be $0.5 \mathrm{MGy}$. Coordinates and structure factors of DgkA have been deposited in the Protein Data Bank (PDB) under accession code $5 \mathrm{dwk}$.

2.1.2. Cas9-RNA-DNA. Crystals of a Cas9-sgRNA-target DNA complex in which the complementary (target) DNA strand was mismatched to the sgRNA guide at positions 1-3 were generated, harvested and cryoprotected as described previously (Anders et al., 2014). Three C2 crystals measuring $\sim 200 \times 100 \times 50 \mu \mathrm{m}$ were used for data collection (Supplementary Fig. S1b). The first, second and third crystals diffracted to $2.2,2.4$ and $2.2 \AA$ resolution, respectively, with anomalous signal extending to $\sim 2.9 \AA$ resolution. The first crystal was large enough to collect two series of multiorientation data sets from two well separated locations on the crystal (Supplementary Fig. S1b, crystal 1). At each location, a total of eight $360^{\circ} \omega$ scans were collected with varying $\chi / \varphi$ settings. For the second crystal, a total of eight $360^{\circ} \omega$ scans were collected at $5^{\circ} \chi$ increments from 0 to $35^{\circ}$ while keeping the $\varphi$ orientation constant. For the third crystal, four $360^{\circ} \omega$ scans were collected at $\varphi=0^{\circ}$ and $\chi=0,10,20$ and $30^{\circ}$, and four $360^{\circ} \omega$ scans were collected at $\varphi=180^{\circ}$ and $\chi=0,10,20$ and $30^{\circ}$ (Table 1). At a data-collection speed of $2 \mathrm{deg} \mathrm{s}^{-1}$, the average dose per $360^{\circ} \omega$ scan was estimated to be $0.25 \mathrm{MGy}$. The coordinates and structure factors of Cas9-RNA-DNA have been deposited in the PDB under accession code $5 \mathrm{fq} 5$. 
2.1.3. $\mathrm{T}_{2} \mathrm{R}$-TTL. Rod-shaped $P 2_{1} 2_{1} 2_{1} \quad \mathrm{~T}_{2} \mathrm{R}$-TTL crystals measuring $\sim 700 \times 100 \times 100 \mu \mathrm{m}$ (Supplementary Fig. S1c) were grown, cryoprotected and snap-cooled in liquid nitrogen as described elsewhere (Prota, Bargsten et al., 2013). Reproducible mounting and cryoprotection was critical as $\mathrm{T}_{2} \mathrm{R}-\mathrm{TTL}$ crystals are extremely sensitive to variation of these conditions (Weinert et al., 2015; PDB entry 4wbn). Of the 20 crystals screened, only three yielded data suitable for merging based on statistical indicators such at $R_{\text {meas }}$ and $\langle I / \sigma(I)\rangle$ (Karplus \& Diederichs, 2015). The three crystals diffracted to $2.3 \AA$ resolution. From each crystal, data were collected at two different positions that were well separated. At the first position, a single high-dose $360^{\circ} \omega$ scan, estimated at $\sim 4 \mathrm{MGy}$,

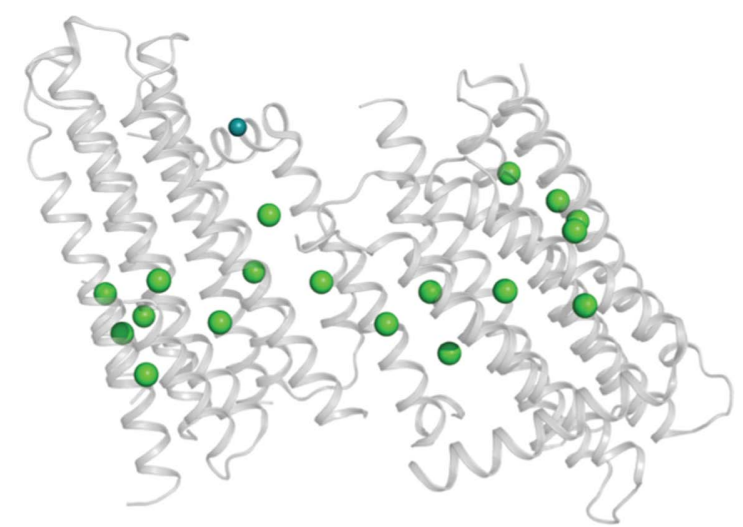

(a)

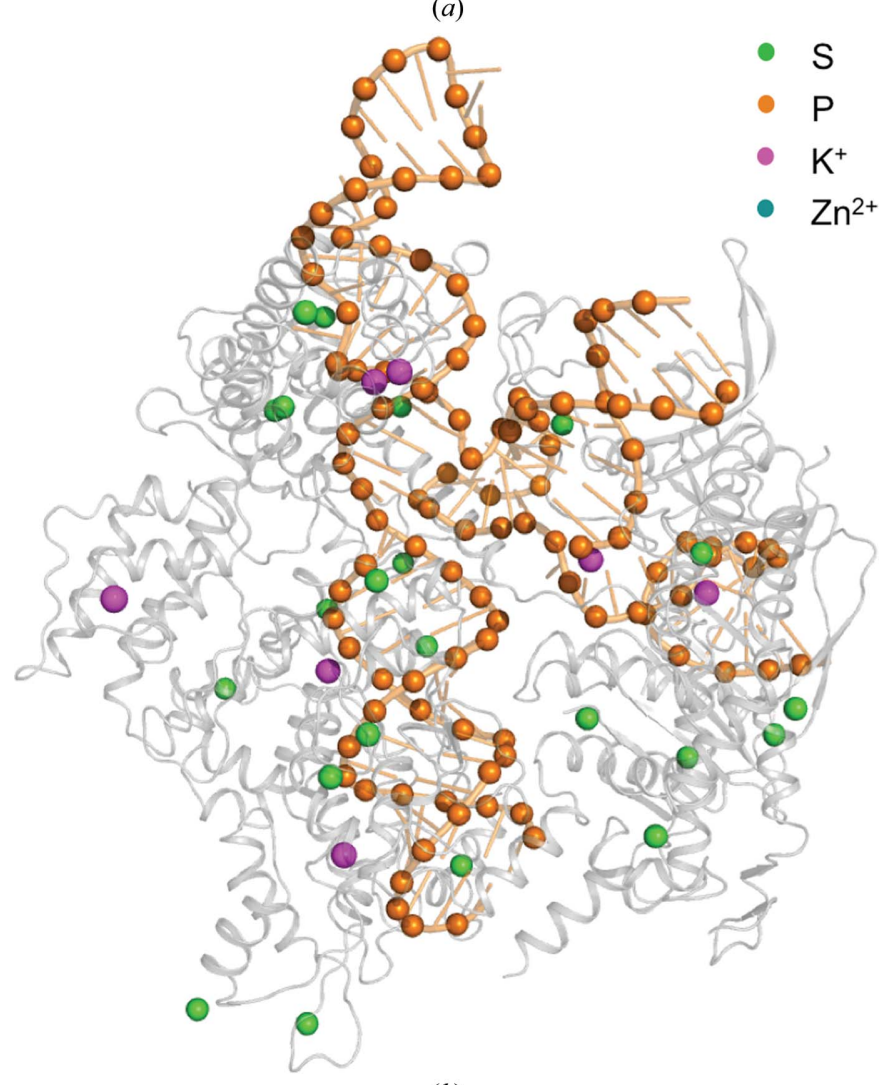

Figure 1

(b)

Native SAD structures. (a) DgkA and (b) Cas9-RNA-DNA. The proteins are shown as grey cartoons and the nucleic acid backbones are coloured orange. Anomalous scatterers are depicted as coloured spheres. was collected (Table 1 and Supplementary Data; $\mathrm{T}_{2} \mathrm{R}$-TTL high dose, low multiplicity, single orientation). At the second position, eight low-dose $720^{\circ} \omega$ scans, corresponding to the same accumulated dose of $\sim 4 \mathrm{MGy}\left(0.25 \mathrm{MGy}\right.$ per $360^{\circ} \omega$ scan), were collected: seven at different $\chi$ increments from 0 to $30^{\circ}$ at constant $\varphi$ and an additional $\omega$ scan at $\chi=10$ or $15^{\circ}$ and $\varphi=90^{\circ}$ (Table 1 and Supplementary Data; $\mathrm{T}_{2} \mathrm{R}$-TTL low dose, high multiplicity, multi-orientation).

\subsection{X-ray fluorescence measurements}

$\mathrm{X}$-ray fluorescence (XRF) analysis was performed on the PHOENIX beamline at the Swiss Light Source at an incident radiation energy of $4.1 \mathrm{keV}$, which is above the Ca $K$ edge $(4.038 \mathrm{keV})$ and not currently accessible at most MX beamlines. The crystal was attached directly to carbon tape and loaded into a $10^{-4} \mathrm{~Pa}$ vacuum chamber. The energy-dispersive $\mathrm{X}$-ray fluorescence spectrum was recorded at room temperature using a single-element solid-state detector (from Roentec) with $180 \mathrm{eV}$ energy resolution. The X-ray beam measuring $100 \times 100 \mu \mathrm{m}$ was directed at the center of the crystal. There was no indication of beam damage over the $120 \mathrm{~s}$ collection time. The XRF spectrum was fitted using PyMca (Solé et al., 2007) to extract the elemental composition of the probed crystal volume. The software provides the elemental composition in terms of single peaks corresponding to different atomic energy-level transitions using Gaussian shape emission lines and energy-dependent photoelectric cross-sections.

\section{Results and discussion \\ 3.1. Native SAD phasing}

3.1.1. DgkA. The integral membrane diacylglycerol kinase DgkA has two trimers in the asymmetric unit representing $6 \times$ 130 residues with a total molecular weight of $84 \mathrm{kDa}$. The substructure has $6 \times 2$ methionines, $6 \times 1$ cysteines and a zinc ion (Fig. 1a), corresponding to a theoretical Bijvoet ratio of $1.1 \%$ at $6 \mathrm{keV}$. Previous SAD and MAD attempts at the $\mathrm{Zn}$ absorption edge were unsuccessful. The structure was originally solved by Se-SAD phasing at the Se peak using crystals diffracting to $2.05 \AA$ resolution (Li et al., 2013). The crystals used in the current study only diffracted to $2.6 \AA$ resolution (see \$2.1.1). Data were collected both with and without multiple crystal orientations (Table 1). Substructure identification using SHELXD was successful with data cut at $4 \AA$ resolution and with $E$ values above 1.5 ; 14 sites out of 19 were identified, 11 of them correctly as determined using SITCOM with reference PDB entry $3 z e 3$ (Li et al., 2013). The single correct solution obtained after 1000 SHELXD attempts had $\mathrm{CC}_{\text {all }}$ and $\mathrm{CC}_{\text {weak }}$ values of 26.2 and 12.0, respectively (Fig. $2 a$ ). The correct hand was identified by density modification in SHELXE. Auto-tracing with a search for $\alpha$-helices resulted in 364 residues (out of 780) built after ten cycles (Fig. 2b), showing a good cross-correlation to the data of $43.95 \%$. The resulting electron-density map (Fig. $3 a$ ) showed a model-map 
cross-correlation of the SHELXE map to the final model of $66.8 \%$.

Analysis of the substructure data exemplifies the weakness of the anomalous data, mainly owing to the disordered Met66 and Cys41 in chains $E$ and $F$. The experimental Bijvoet ratio of $1 \%$ for this sample was therefore lower than the expected theoretical value of $1.1 \%$.

3.1.2. Cas9-RNA-DNA. The CRISPR-associated protein Cas9 is an RNA-guided endonuclease that has been repurposed for genome editing and gene-expression control. The Cas9-RNA-DNA complex, which crystallizes in the lowsymmetry space group $C 2$, was initially solved with SAD phases obtained from selenomethionine and iridium derivatives (Anders et al., 2014). For native SAD phasing experiments, we used crystals of the Cas9-sgRNA-target DNA complex in which the complementary DNA strand contains mismatches to the guide RNA at positions 1-3 (see §2.1.2). The asymmetric unit contains 1371 amino acids, 83 RNA nucleotides and 39 DNA nucleotides (total molecular weight $\sim 200 \mathrm{kDa}$ ), with $24 \mathrm{~S}$ atoms and $120 \mathrm{P}$ atoms (Fig. 1b), which comprises the largest substructure solved with native SAD phasing to date. In this case, data were collected from three native crystals diffracting to $\sim 2.2 \AA$ resolution in multiple orientations at a wavelength of $2.066 \AA$ and the data were

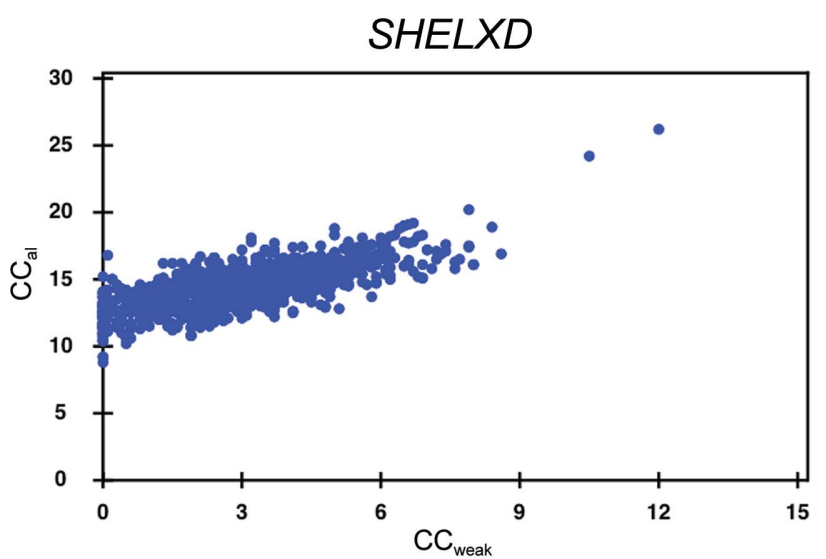

(a)

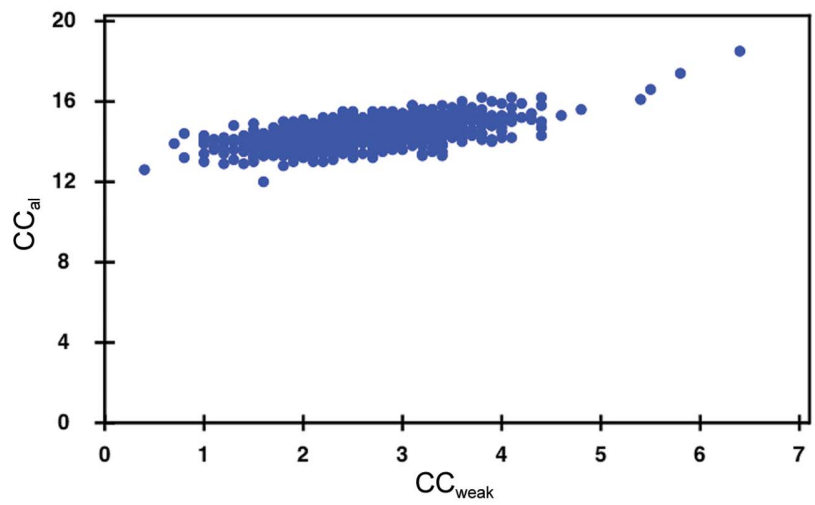

(c)

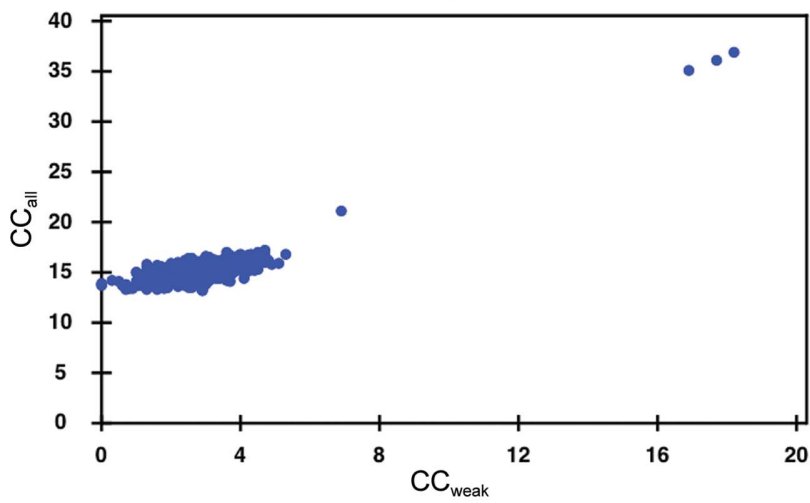

(e)

Figure 2

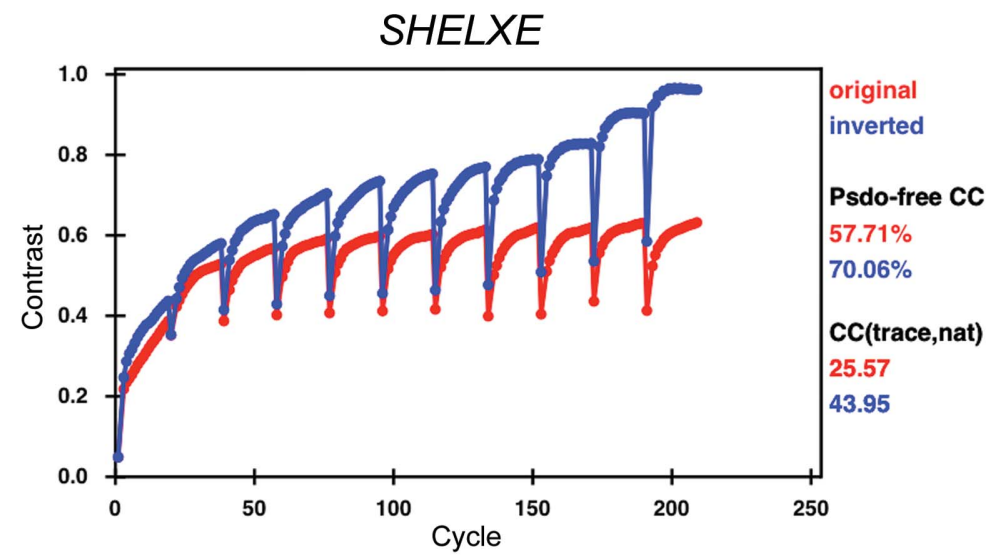

(b)

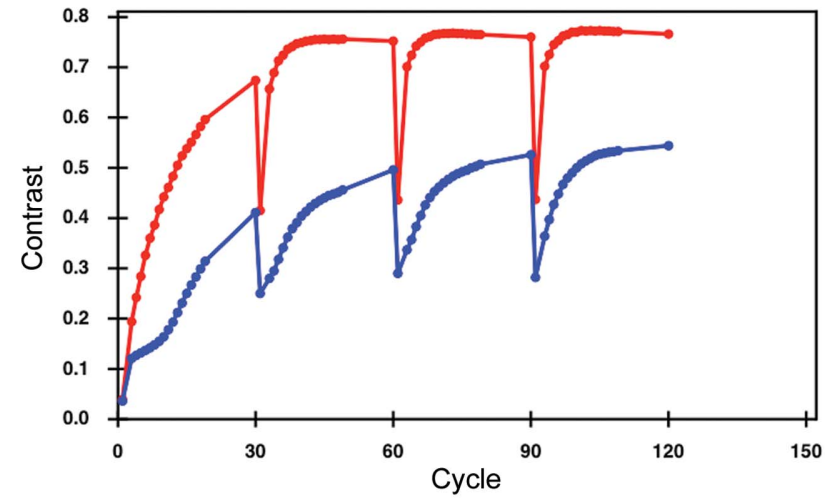

$(d)$

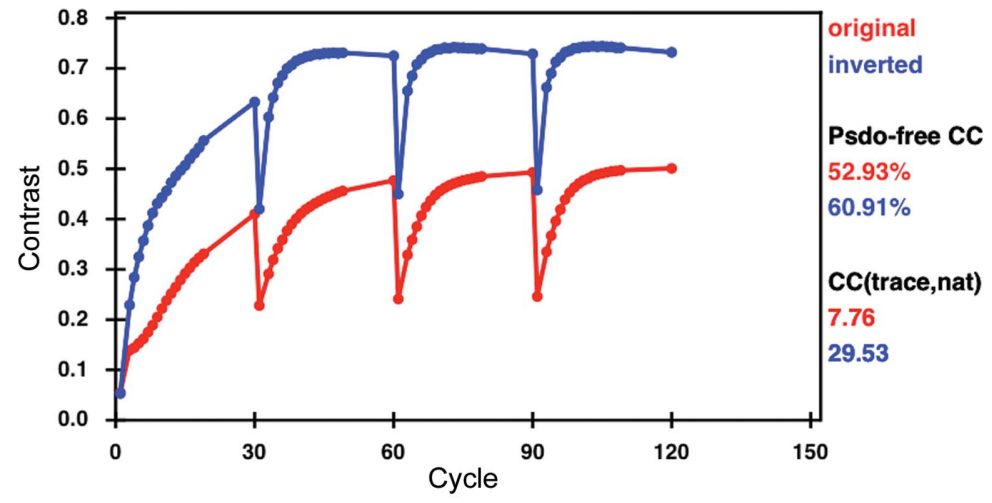

(f)

SHELX output. (a) SHELXD substructure-determination output for DgkA for 1000 trials. (b) SHELXE output for DgkA after ten cycles of autotracing. (c) SHELXD substructure-determination output for Cas9 for 1000 trials. (d) SHELXE output for Cas9 after three cycles of auto-tracing. (e) SHELXD substructure determination for $\mathrm{T}_{2}$ R-TTL with 1000 trials. $(f)$ Three cycles of chain tracing with phasing and density modification in $S H E L X E$ for $\mathrm{T}_{2} \mathrm{R}$-TTL. 
merged in an initial attempt at locating sulfur and phosphorus positions from the anomalous peaks (Table 1). The substructure solution could be obtained with 1000 SHELXD trials using a high-resolution cutoff of $2.6 \AA$ in a search for 65 sites, excluding data with $E$ values below 1.2 (Fig. $2 c$ ). The program initially identified 45 correct sites out of a total of 150 as determined using SITCOM with reference PDB entry 4un5 (Anders et al., 2014). SHELXE substructure refinement completed the substructure to 114 sites, resulting in a very clear hand separation (Fig. $2 d$ ). Three cycles of chain tracing with a search for $\alpha$-helices resulted in a readily interpretable map (Fig. 3b) building a $\mathrm{C}_{\alpha}$ chain of 1060 residues (out of 1372) after three cycles (Fig. $2 b$ ) with a cross-correlation of $28.75 \%$ to the data. The model-map cross-correlation of the SHELXE map to the final model was $68.0 \%$.

Substructure determination was only successful using data merged from three crystals collected in multiple orientations. The increase in the anomalous peak height resulting from the merging of new data sets (coming from either new orientations of the same crystal or from additional crystals) is shown in Fig. 4. We limited the accumulated dose on each crystal (or crystal location) to a conservative $\sim 2 \mathrm{MGy}$ in order to minimize the effects of radiation damage. Additional dose brought little additional information; in the case of crystal 1, we

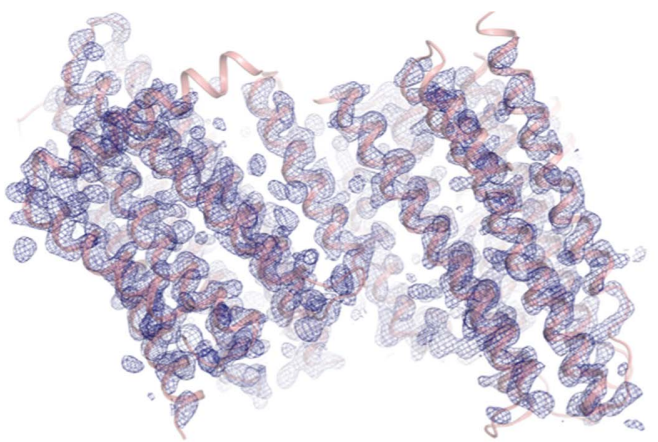

(a)

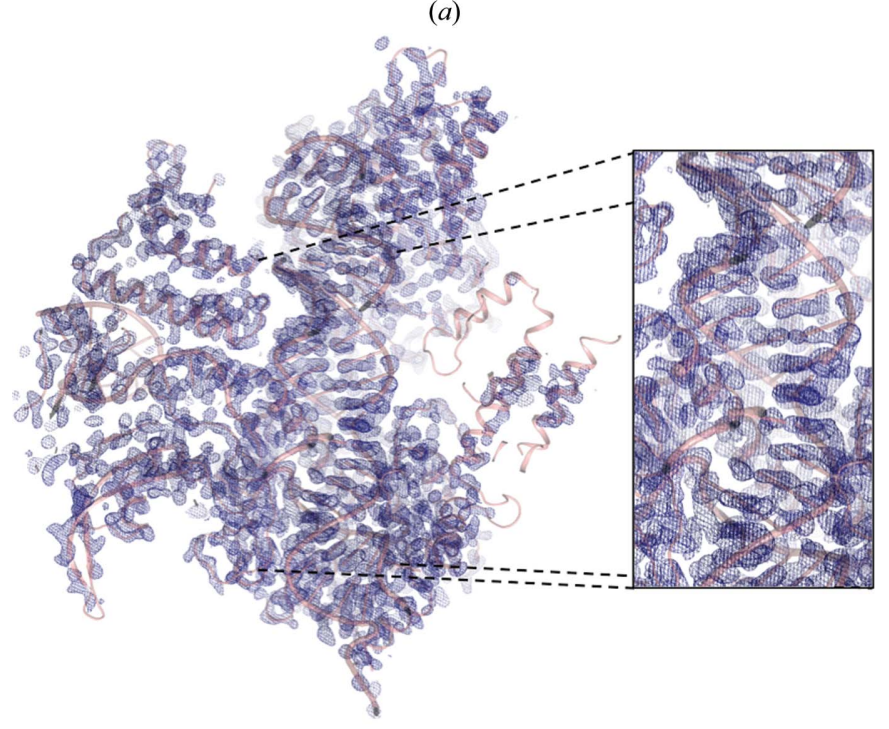

(b)

Figure 3

Experimental maps after SHELXE phasing and density modification, contoured at $2 \sigma$. (a) DgkA map. (b) Cas9-RNA-DNA map. observed that the gain in anomalous signal was small beyond 2 MGy and plateaued at 4 MGy (Supplementary Fig. S2).

\subsection{X-ray fluorescence analysis}

Assigning certain atoms, especially light monoatomic ions, in a crystal structure can be difficult. An added benefit of data collection at low energy is that the anomalous scattering from some of these elements can aid in their correct assignment (Mueller-Dieckmann et al., 2007). In the case of the Cas9sgRNA-DNA complex, certain ions originally modelled as $\mathrm{Mg}^{2+}$ (PDB entry 4un5) displayed strong anomalous signal $(>10 \sigma)$, suggesting alternative atom assignments. However, it was not possible to unambiguously identify the corresponding element from the anomalous peak heights or from an examination of the chemical architecture of the site. One of the crystals was subsequently used to collect a fluorescence spectrum at an incident energy of $4.1 \mathrm{keV}$ (see §2.2). The spectrum confirmed the presence of $\mathrm{K}^{+}$(Fig. 5), which was likely to arise from the crystallization condition (the buffer solution contained $250 \mathrm{mM} \mathrm{KCl}$ and $300 \mathrm{~m} M \mathrm{KSCN})$. We therefore reassigned six $\mathrm{Mg}^{2+}$ ions as $\mathrm{K}^{+}$ions and found an additional one. Subsequent refinement of the anomalous scattering contribution $f^{\prime \prime}$, as implemented in phenix.refine (Echols et al., 2014), gave $f^{\prime \prime}\left(\mathrm{K}^{+}\right)$values between 1.58 and $1.72 \mathrm{e}$, in agreement with the theoretical value of $1.77 \mathrm{e}$.

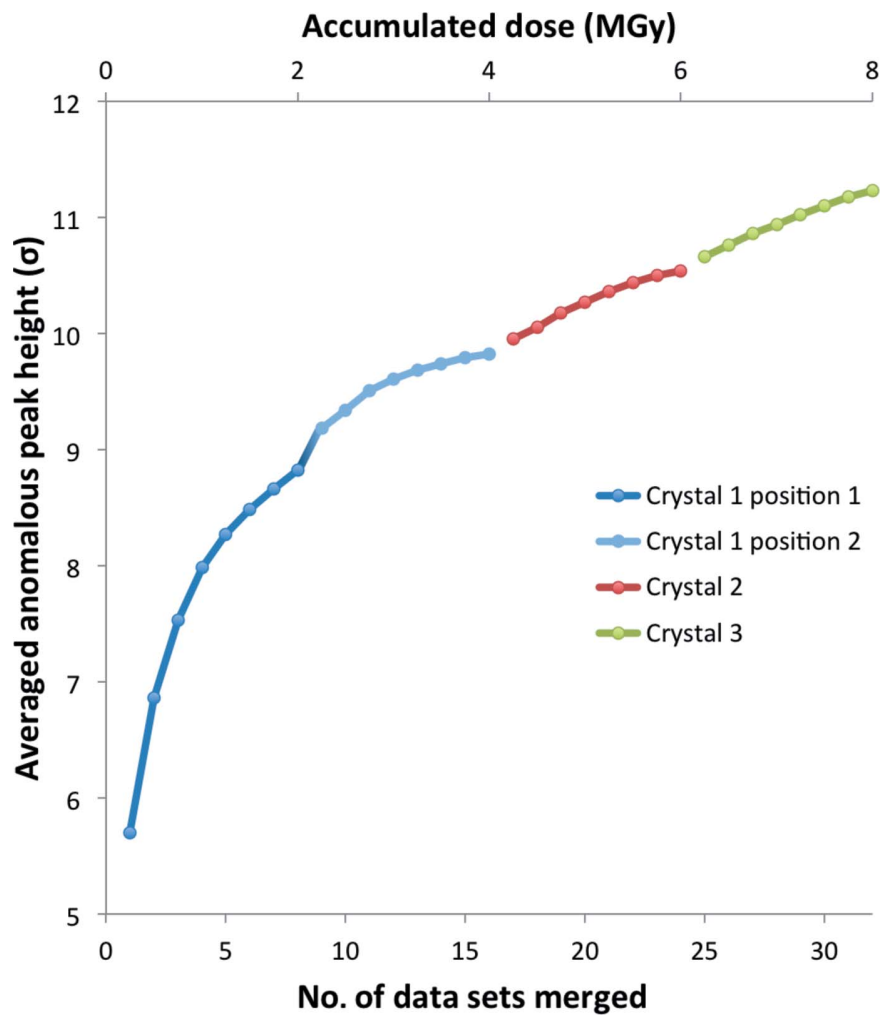

Figure 4

Averaged anomalous peak height of the first 70 anomalous peaks for Cas9-RNA-DNA merged data sets. A total of $3 \times 360^{\circ}$ data sets were merged from three different crystals. Data from the first crystal were collected at two well separated positions (Supplementary Fig. S1b, crystal $1)$. The X-ray dose (upper $x$ axis) accumulated at each position was around $2 \mathrm{MGy}$. 
3.3. Optimal data-collection strategy for challenging native SAD phasing: $T_{2} R-T T L$ as a test case

$\mathrm{T}_{2} \mathrm{R}$-TTL is a multiprotein/multiligand complex composed of tubulin (T), the microtubule destabilizing- and stathminlike protein RB3 and the tubulin-modifying enzyme tubulin tyrosine ligase (TTL; Prota, Magiera et al., 2013). The asymmetric unit, composed of two $\alpha$-tubulin, two $\beta$-tubulin, one RB3, one TTL, two GTP, two GDP and one AMPPNP molecules, consists of 2317 amino acids (total molecular weight of $\sim 266 \mathrm{kDa}$ ) and has a substructure with $118 \mathrm{~S}, 13 \mathrm{P}$, three $\mathrm{Ca}$ and two $\mathrm{Cl}$ sites. Following our previous successful structure solution of $\mathrm{T}_{2} \mathrm{R}$-TTL by native SAD phasing using a single crystal, multi-orientation data-collection strategy (Weinert et al., 2015), we decided to use this system to evaluate the benefits of the combined multi-orientation, multi-crystal strategy. $\mathrm{T}_{2} \mathrm{R}$-TTL is an excellent example of a challenging test case; it has a large asymmetric unit and a large number of scatterers and, as shown below, it forms crystals of variable quality.

Using multiple native SAD data sets measured from a single $\mathrm{T}_{2} \mathrm{R}$-TTL crystal (see $\$ 2.1 .3$ and Supplementary Data), it was not possible to determine the substructure. This is likely because the quality of the crystals used in the current work was less than that observed in the original study (Weinert et al., 2015). A substructure solution with SHELXD could only be obtained upon merging data sets from three different crystals each measured in eight different orientations (Table 1 and Supplementary Data) in a search for 75 sites using a resolution cutoff of $3.3 \AA$ and with $E$ values above 1.5 (Fig. $2 e$ ). The successful SHELXD substructure solution had a $\mathrm{CC}_{\mathrm{all}}$ and $\mathrm{CC}_{\mathrm{weak}}$ of 36.9 and 18.2 , respectively. The number of correct sites, as determined using SITCOM with reference PDB entry 4wbn, was 86 and this increased to 111 using substructure refinement in SHELXE. Density modification resulted in a clear separation of hands (Fig. $2 f$ ). Three cycles of chain tracing with a search for $\alpha$-helices resulted in a readily

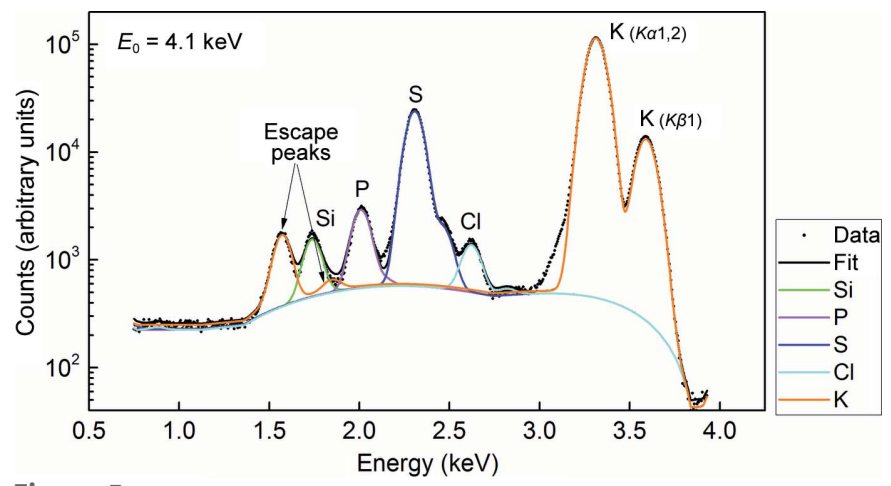

Figure 5

Log scale of the X-ray fluorescence spectrum of a Cas9-RNA-DNA crystal recorded at an incident energy of $4.1 \mathrm{keV}$. The measured spectrum is plotted as black dots and the fit as black (total) and coloured (for different elements) lines. The main contributions (orange) originate from the potassium $K \alpha 1,2\left(K-L_{\mathrm{III}}\right.$ and $\left.K-L_{\mathrm{II}}\right)$ and $K \beta 1\left(K-M_{\mathrm{III}}\right)$ characteristic lines. The smaller (orange) peaks between 1.5 and $2 \mathrm{keV}$ represent the $\mathrm{K}-$ $\mathrm{Si}$ escape peaks and are an artifact of the measurement (Papp \& Campbell, 2001). The Si peak (green line) originates from the crystal holder. interpretable map, building a $\mathrm{C}_{\alpha}$ chain of 1669 residues (out of 2319) after three cycles with a cross-correlation of $28.75 \%$ to the data. The model-map cross-correlation of the SHELXE map to the final model was $78.5 \%$.

The merit of combining low-dose data from multiple crystals in multiple orientations, which was readily observed in the merging statistics (see Supplementary Data), was assessed by comparing the average anomalous peak heights for four datacollection strategies: (i) high dose, single orientation from a single crystal; (ii) low dose, multiple orientation from a single crystal; (iii) merging high-dose, single-orientation data from three crystals; and (iv) merging low-dose, multiple-orientation data from three crystals (Fig. 6). A single-orientation scan at high dose gave only 23 anomalous peaks above $10 \sigma$, which was insufficient for successful substructure solution. Despite a threefold higher accumulated dose, merging high-dose data from three different crystals resulted in 50 anomalous sites above $10 \sigma$, similar to merging low-dose, multiple-orientation data from a single crystal. However, this was still insufficient for successful substructure solution. The substructure search was only successful when low-dose, multiple-orientation data sets from three crystals were merged, yielding 70 anomalous sites above $10 \sigma$. This is consistent with our previous study, in which substructure solution and hence native SAD phasing were successful by merging low-dose, multiple-orientation scans from a single crystal, giving 80 anomalous sites above $10 \sigma$ (Weinert et al., 2015). This is also consistent with a study showing that substructure searches tend to be successful when mean peak heights in the anomalous difference map are above

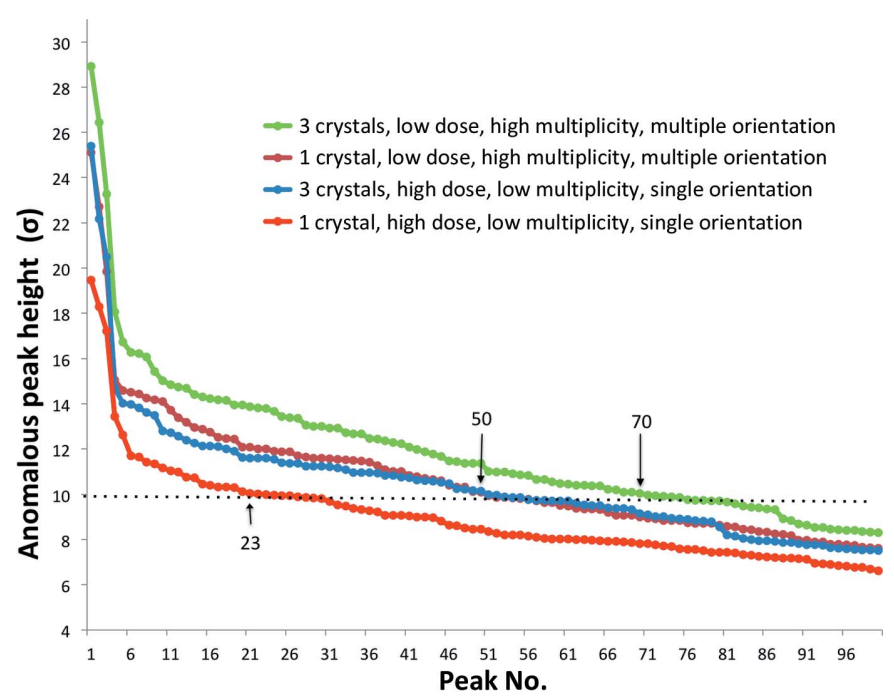

Figure 6

Comparison of anomalous peak heights for various data-collection strategies. The anomalous peak heights for the first 100 peaks are shown for four data-collection strategies for $\mathrm{T}_{2} \mathrm{R}$-TTL data sets: (i) high dose, low multiplicity, single orientation at a single crystal location (red), (ii) low dose, high multiplicity, multiple orientation at a single crystal location (brick), (iii) merging high-dose, single-orientation data from three crystals (blue) and (iv) merging low-dose, multiple-orientation data from three crystals (green). For each crystal, data were collected with the same dose at two well separated positions. The dotted line marks the $10 \sigma$ threshold. The arrows point to the last peak above $10 \sigma$ and show the number of sites above this threshold. 
a certain threshold (Bunkóczi et al., 2015); for $\mathrm{T}_{2} \mathrm{R}$-TTL crystals, this threshold is around $10 \sigma$ (dashed line in Fig. 6).

Taken together, there is a clear benefit in combining the two data-collection strategies: (i) a low-dose, multiple-orientation approach efficiently extracts as much anomalous data as possible while minimizing radiation damage and (ii) if the anomalous signal from a single crystal sample is not sufficient for successful phasing, data sets from multiple statistically equivalent crystals can be merged. Combining the two approaches means that fewer crystal samples are required. In addition, for the same accumulated dose, there is no difference in diffraction resolution between data sets collected using a high-dose, single-orientation strategy and merged data sets collected using a low-dose, multiple-orientation strategy (Table 1 and Supplementary Data).

\section{Conclusion and outlook}

Native SAD could be considered the 'ideal' de novo phasing method for macromolecules because it relies solely on information from atoms that are naturally present in the sample. The method is readily applicable to challenging cases. Accordingly, there is every reason to try native SAD first with macromolecules that contain a sufficient number of naturally occurring anomalous scatterers. Here, we show that multicrystal averaging of data sets used in conjunction with a lowdose, multi-orientation strategy is beneficial in difficult cases where data from one crystal are insufficient. Further, low-dose data collection in multiple orientations means that fewer crystal specimens are needed to provide the required data accuracy.

That said, this combined approach is still in its infancy and there is room for improvement in order to make native SAD more broadly applicable and more generally accepted, especially in cases of low resolution, which are currently dominated by heavy-atom phasing methods. In addition, collecting multiple $360^{\circ}$ scans on multiple crystals, as detailed here, takes more time than single-orientation high-dose scans and may be unappealing in situations where synchrotron beamtime is limited. Nevertheless, advances in data-acquisition hardware, in particular detector instrumentation, will rapidly improve the situation. Furthermore, the method described here lends itself to automation.

As the frontiers of crystallography continue to expand, fundamental techniques for solving the phase problem will continue to evolve. In particular, new methods such as serial crystallography have presented new challenges that require the development of tools for obtaining the most accurate measurements. Data collection at energies below $6 \mathrm{keV}$ should boost the anomalous signal, but presents technical challenges, especially air absorption and scattering, sample absorption and detector geometry. These are currently being investigated at operational beamlines such as BL-1A at the Photon Factory (see Rose et al., 2015), as well as upcoming beamlines such as I23 at Diamond Light Source (Allan et al., 2015) and LAX at NSLS-II (Hendrickson, 2014).

\section{Acknowledgements}

This work was supported in part by funding from Science Foundation Ireland (12/IA/1255 to MC), by a grant from the Swiss National Science Foundation (310030B_138659 to MOS) and a European Research Council Starting Grant (ANTIVIRNA, Grant no. 337284 to MJ).

\section{References}

Adams, P. D. et al. (2010). Acta Cryst. D66, 213-221.

Akey, D. L., Brown, W. C., Konwerski, J. R., Ogata, C. M. \& Smith, J. L. (2014). Acta Cryst. D70, 2719-2729.

Allan, D. R., Collins, S. P., Evans, G., Hall, D., McAuley, K., Owen, R. L., Sorensen, T., Tang, C. C., von Delft, F., Wagner, A. \& Wilhelm, H. (2015). Eur. Phys. J. Plus, 130, 56.

Anders, C., Niewoehner, O., Duerst, A. \& Jinek, M. (2014). Nature (London), 513, 569-573.

Berman, H. M., Westbrook, J., Feng, Z., Gilliland, G., Bhat, T. N., Weissig, H., Shindyalov, I. N. \& Bourne, P. E. (2000). Nucleic Acids Res. 28, 235-242.

Brockhauser, S., Ravelli, R. B. G. \& McCarthy, A. A. (2013). Acta Cryst. D69, 1241-1251.

Bunkóczi, G., McCoy, A. J., Echols, N., Grosse-Kunstleve, R. W., Adams, P. D., Holton, J. M., Read, R. J. \& Terwilliger, T. C. (2015). Nature Methods, 12, 127-130.

Dall'Antonia, F. \& Schneider, T. R. (2006). J. Appl. Cryst. 39, 618-619.

Debreczeni, J. É., Bunkóczi, G., Ma, Q., Blaser, H. \& Sheldrick, G. M. (2003). Acta Cryst. D59, 688-696.

DeLano, W. L. (2002). PyMOL. http://www.pymol.org.

Echols, N., Morshed, N., Afonine, P. V., McCoy, A. J., Miller, M. D., Read, R. J., Richardson, J. S., Terwilliger, T. C. \& Adams, P. D. (2014). Acta Cryst. D70, 1104-1114.

El Omari, K., Iourin, O., Kadlec, J., Fearn, R., Hall, D. R., Harlos, K., Grimes, J. M. \& Stuart, D. I. (2014). Acta Cryst. D70, 2197-2203.

Finke, A. D., Panepucci, E., Vonrhein, C., Wang, M., Bricogne, G. \& Oliéric, V. (2016). Methods Mol. Biol. 1320, 175-191.

Hendrickson, W. A. (2014). Q. Rev. Biophys. 47, 49-93.

Hendrickson, W. A. \& Teeter, M. M. (1981). Nature (London), 290, 107-113.

Henrich, B., Bergamaschi, A., Broennimann, C., Dinapoli, R., Eikenberry, E. F., Johnson, I., Kobas, M., Kraft, P., Mozzanica, A. \& Schmitt, B. (2009). Nucl. Instrum. Methods Phys. Res. A, 607, 247-249.

Huang, C.-Y., Olieric, V., Ma, P., Panepucci, E., Diederichs, K., Wang, M. \& Caffrey, M. (2015). Acta Cryst. D71, 1238-1256.

Kabsch, W. (2010). Acta Cryst. D66, 125-132.

Karplus, P. A. \& Diederichs, K. (2012). Science, 336, 1030-1033.

Karplus, P. A. \& Diederichs, K. (2015). Curr. Opin. Struct. Biol. 34, 60-68.

Klinke, S., Foos, N., Rinaldi, J. J., Paris, G., Goldbaum, F. A., Legrand, P., Guimarães, B. G. \& Thompson, A. (2015). Acta Cryst. D71, 1433-1443.

Li, D., Lyons, J. A., Pye, V. E., Vogeley, L., Aragão, D., Kenyon, C. P., Shah, S. T. A., Doherty, C., Aherne, M. \& Caffrey, M. (2013). Nature (London), 497, 521-524.

Liu, Q., Dahmane, T., Zhang, Z., Assur, Z., Brasch, J., Shapiro, L., Mancia, F. \& Hendrickson, W. A. (2012). Science, 336, 1033-1037. Liu, Q., Guo, Y., Chang, Y., Cai, Z., Assur, Z., Mancia, F., Greene, M. I. \& Hendrickson, W. A. (2014). Acta Cryst. D70, 2544-2557.

Liu, Q., Liu, Q. \& Hendrickson, W. A. (2013). Acta Cryst. D69, 1314 1332.

Mueller-Dieckmann, C., Panjikar, S., Schmidt, A., Mueller, S., Kuper, J., Geerlof, A., Wilmanns, M., Singh, R. K., Tucker, P. A. \& Weiss, M. S. (2007). Acta Cryst. D63, 366-380. 
Mueller-Dieckmann, C., Panjikar, S., Tucker, P. A. \& Weiss, M. S. (2005). Acta Cryst. D61, 1263-1272.

Pape, T. \& Schneider, T. R. (2004). J. Appl. Cryst. 37, 843-844.

Papp, T. \& Campbell, J. L. (2001). X-ray Spectrom. 30, 77-82.

Prota, A. E., Bargsten, K., Zurwerra, D., Field, J. J., Díaz, J. F., Altmann, K.-H. \& Steinmetz, M. O. (2013). Science, 339, 587-590.

Prota, A. E., Magiera, M. M., Kuijpers, M., Bargsten, K., Frey, D., Wieser, M., Jaussi, R., Hoogenraad, C. C., Kammerer, R. A., Janke, C. \& Steinmetz, M. O. (2013). J. Cell Biol. 200, 259-270.

Rose, J. P., Wang, B.-C. \& Weiss, M. S. (2015). IUCrJ, 2, 431-440.

Sheldrick, G. M. (2010). Acta Cryst. D66, 479-485.
Solé, V. A., Papillon, E., Cotte, M., Walter, P. \& Susini, J. (2007). At. Spectrosc. 62, 63-68.

Thorn, A. \& Sheldrick, G. M. (2011). J. Appl. Cryst. 44, 12851287.

Waltersperger, S., Olieric, V., Pradervand, C., Glettig, W., Salathe, M., Fuchs, M. R., Curtin, A., Wang, X., Ebner, S., Panepucci, E., Weinert, T., Schulze-Briese, C. \& Wang, M. (2015). J. Synchrotron Rad. 22, 895-900.

Weinert, T. et al. (2015). Nature Methods, 12, 131-133.

Zeldin, O. B., Gerstel, M. \& Garman, E. F. (2013). J. Appl. Cryst. 46, 1225-1230. 